\title{
Millimeter-Wave Spectroscopy of Weakly Bound Molecular Complexes and Small Clusters
}

\author{
L. A. Surin ${ }^{1,2}$ \\ ${ }^{1}$ Institute of Spectroscopy, Troitsk, Moscow, Russia, surin@isan.troitsk.ru \\ ${ }^{2}$ I. Physikalisches Institut, University of Cologne, Cologne, Germany
}

Currently, there are a number of international projects on a full-scale study of the infrared and submillimeter-wave (SMMW) radiation in space, such as the Space Observatory "Herschel” (mission is completed, analysis of the data is in progress), the Stratospheric Observatory "SOFIA" based on a Boeing-747SP wide-body aircraft, astronomical interferometer of MMW and SMMW telescopes in the Atacama Desert of northern Chile "ALMA", and many others. The demand of high-level spectroscopic data, including the parameters of interaction between abundant in the interstellar space molecules and atoms, is extremely high, because it is necessary for the interpretation of the information coming from the radio telescopes. Many laboratory and theoretical research directed on the understanding of the interaction between ammonia $\left(\mathrm{NH}_{3}\right)$, water $\left(\mathrm{H}_{2} \mathrm{O}\right)$, carbon monoxide $(\mathrm{CO})$, nitrogen $\left(\mathrm{N}_{2}\right)$, etc. and their main partners by the collisions in the interstellar medium - molecular hydrogen $\mathrm{H}_{2}$ and atomic helium He [1, 2]. These data are then used to test intermolecular interaction potentials, and they provide the necessary background for the development for the astrophysical models.

Another application, in which the intermolecular interactions with $\mathrm{He}$ and $\mathrm{H}_{2}$ play an important role, is the doping of helium or para-hydrogen clusters with a probe molecule to perform high resolution spectroscopy. Such experiments are directed on the detection of superfluid response of molecular rotation in the $\mathrm{He}$ and para $-\mathrm{H}_{2}$ clusters [3].

Typically, microwave (MW) spectra provide information about ground state end-over-end pure rotational energy levels of the complexes, MMW spectra give access to their internal rotor states, and SMMW or $\mathrm{THz}$ wavelengths probe intermolecular vibrations. Gas phase high resolution spectroscopic data give unambiguous information on the dynamics, composition, and structure of the complexes, because their bound states are very sensitive to the interaction potential.

The overview of the spectroscopic methods as well as recent results on molecular complexes and small clusters are presented.

\section{OROTRON Spectrometer with Supersonic Jet}

In OROTRON, the MMW radiation is produced by interaction of an electron beam with the electromagnetic field of an open Fabry-Perot resonator. The principal scheme of the spectrometer is shown in Fig. 1. In order to allow the measurements in the molecular jet expansion, the entire OROTRON is placed into a vacuum chamber. The high vacuum part of the OROTRON includes the cathode, the collector and the fixed plane mirror with a periodic structure ruled on its surface. This part is separated from the rest of the resonator by a thin mica window, which is transparent to the MMW radiation. The second part of the OROTRON consists of a movable spherical mirror. These two parts are connected to each other by a hollow brass cylinder with two openings for the propagation of a molecular jet through the resonator.

Absorption inside the resonator is sensitively detected by measuring the appropriate change of the electron current in the collector circuit. The principle of the method is that a change in the quality factor of the resonator due to the absorption in the gas will cause a change in the velocity distribution of the electrons and thus in their passage to the collector [4].

The double resonance option strongly helps in the identification of complex spectra. If one of the two transitions in a three level system is saturated, the populations of the levels and, correspondingly, the radiation absorption intensity in the second transition change. Therefore, the absorption of radiation from an additional source in the first transition can be detected in a change in the signal of the spectrometer tuned to the second transition. As shown in Fig. 1, the OROTRON spectrometer assumes the role of signal detection, while the microwave pump radiation is delivered by a microwave synthesizer followed by a high power broadband amplifier (2-110 GHz). The molecular beam travels perpendicular to both MMW and MW radiation beams and interacts simultaneously with them.

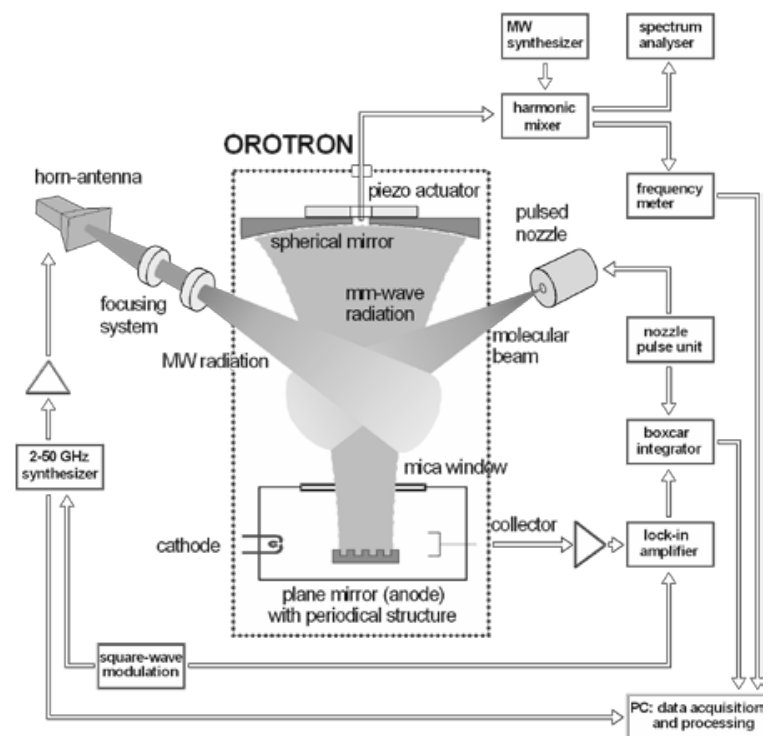

Fig. 1. Schematic diagram of the OROTRON jet spectrometer (100-150 GHz) with MW-MMW double resonance arrangement. 
This technique was applied to study many weakly bound van der Waals systems [5]. Fig. 2 shows one example of a $20 \mathrm{MHz}$ survey for $R(0)$ transitions of the $\mathrm{He}_{8}-{ }^{12} \mathrm{C}^{18} \mathrm{O}$ cluster [3].

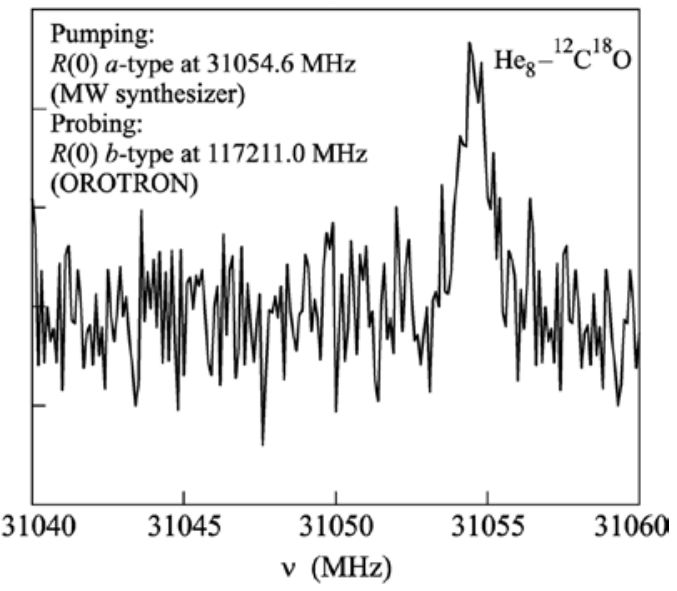

Fig. 2. Record of the $R(0)$ transition in the $\mathrm{He}_{8}-{ }^{12} \mathrm{C}^{18} \mathrm{O}$ cluster by the double resonance method with OROTRON.

\section{Supersonic Jet Spectrometer for Terahertz Applctions (SuJeSTA)}

For measurements of the weakly bound van der Waals $\mathrm{NH}_{3}-\mathrm{H}_{2}$ complexes the SuJeSTA spectrometer (150-800 GHz) [6] was applied. Details of the current experimental setup used in the Astrophysics group of the University of Kassel for carbon clusters experiments are shown in Fig. 3. A microwave synthesizer is used as a radiation source, followed by a frequency multiplier. The SMMW radiation intersects a pulsed supersonic jet $\sim 5 \mathrm{~cm}$ downstream in a perpendicular configuration.

A multi-pass optics is used to further enhance the signal-to-noise ratio. The absorption signal is detected by a low-noise liquid-He cooled hot electron bolometer. The frequency of the synthesizer is modulated at $40 \mathrm{kHz}$, and phase sensitive detection of the signal is achieved by a lock-in amplifier in $2 f$-mode operation. In addition to the frequency modulation of the radiation source, an on-off modulation of the jet is used for background subtraction through a pair of boxcar integrators. The combination of frequency and source modulation substantially improves the $\mathrm{S} / \mathrm{N}$ ratio and at the same time suppresses standing wave effects.

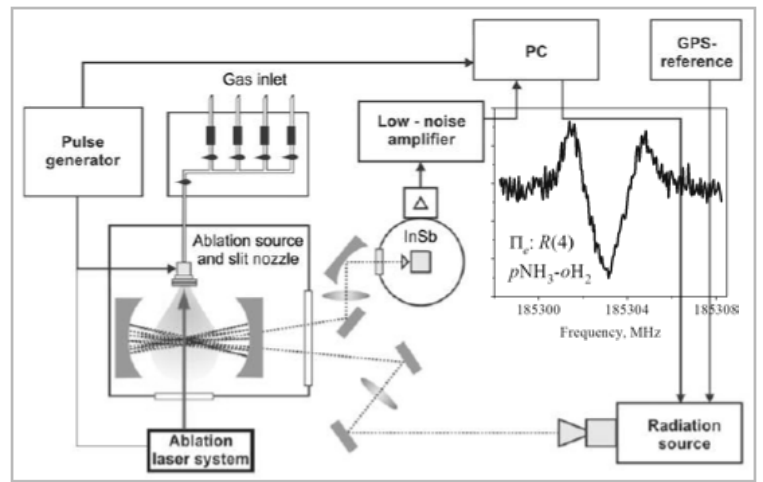

Fig. 3. SuJeSTA spectrometer (150-800 GHz) with multipass cell and recorded line of the $\mathrm{pNH}_{3}-\mathrm{OH}_{2}$ complex.

\section{Double Resonance Spectroscopy with Cryogenic Ion Trap Apparatus}

A novel action spectroscopic method for rotational spectroscopy of weakly bound complexes, which has been recently developed in the Spectroscopy group of the University of Cologne. This method uses a double resonance consisting of a rotational transition (MMW) followed by a predissociating transition (IR), and uses the final destruction of the complex as the spectroscopic action signal. This approach was applied to obtain high-resolution rotational data of the $\mathrm{He}-\mathrm{HCO}^{+}$complex. $\mathrm{HCO}^{+}$is particularly interesting because it is probably the most abundant molecular ion in dense cores of interstellar molecular clouds and its complex with He is regarded as reaction intermediate of the proton exchange reaction.

The experiments have been carried out in the 22pole ion trap machine COLTRAP [7]. The spectroscopic parameters of the ground state have been determined to microwave precision, thus providing the most reliable reference data for testing available He$\mathrm{HCO}^{+}$interaction potentials.

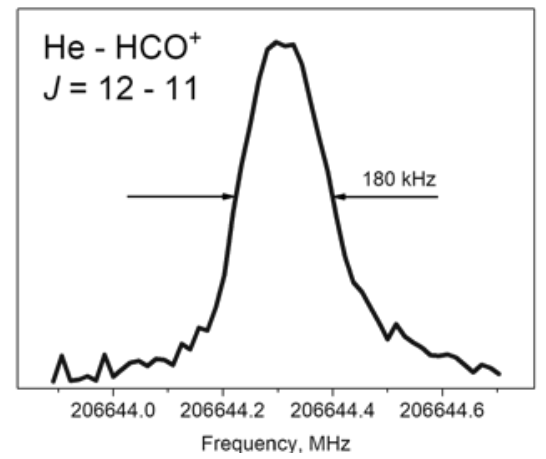

Fig. 4. Example for the double resonance technique applied to the $\mathrm{He}-\mathrm{HCO}^{+}$complex.

The author acknowledges the Russian Science Foundation (Grant 17-12-01395).

\section{References}

1. Green S., Thaddeus P. Rotational excitation of CO by collisions with $\mathrm{He}, \mathrm{H}$, and $\mathrm{H}_{2}$, under conditions in interstellar clouds // Astrophys. J. 1976. V. 205, P. 766-785.

2. Chefdeville S., Stoecklin T., Naulin C., et. al. Experimental and theoretical analysis of low-energy $\mathrm{CO}+\mathrm{H}_{2}$ inelastic collisions // Astrophys. J. Lett. 2015. V. 799, L9.

3. Surin $L$. Nonclassical rotations of single molecule in small helium and hydrogen clusters: manifestation of "microscopic superfluidity" // JETP Lett. 2013. V. 97, P. 57-65.

4. Surin L.A., Dumesh B.S., Lewen F., Roth D.A., et. al. Millimeter-wave intracavity-jet OROTRON-spectrometer for investigation of van der Waals complexes // Rev. Sci. Instrum. 2001. V. 72, P. 2535-2542.

5. Dumesh B.S., Surin L.A. Unusual rotations in helium and hydrogen nanoclusters and "nanoscopic" superfluidity // Physics-Uspekhi. 2006. V. 49, P. 1113-1129.

6. Surin L.A., Tarabukin I.V., Schlemmer S., Breier A.A., Giesen T. F., et. al. Rotational spectroscopy of the $\mathrm{NH}_{3}-\mathrm{H}_{2}$ complex // Astrophys. J. 2017. V. 838, Art. 27.

7. Salomon T., Töpfer M., Schreier P., Schlemmer S., Asvany O., Kohguchi H., Surin L. Double Resonance Rotational Spectroscopy of $\mathrm{He}-\mathrm{HCO}^{+} / /$Phys. Chem. Chem. Phys. 2018. 